\title{
Geographic Diversity in Atypical Hemolytic Uremic Syndrome (aHUS): The Genetic Background of aHUS Cohort in Japan
}

\author{
Yoichiro Ikeda*, Yoko Yoshida, Yuuka Sugawara, Masaomi Nangaku
}

Division of Nephrology and Endocrinology, The University of Tokyo, 7-3-1, Hongo, Bunkyo-ku, Tokyo, 113-8655, Japan

\section{Article Info}

\section{Article Notes}

Received: July 09, 2018

Accepted: August 03, 2018

\section{${ }^{*}$ Correspondence:}

Dr. Yoichiro Ikeda, Division of Nephrology and

Endocrinology, The University of Tokyo, 7-3-1, Hongo,

Bunkyo-ku, Tokyo, 113-8655, Japan;

Email: ikeda-tky@umin.ac.jp

(c) $2018 \mathrm{lkeda}$ Y. This article is distributed under the terms of the Creative Commons Attribution 4.0 International License.
Abstract

Atypical hemolytic uremic syndrome (aHUS) is a rare disease caused by the dysfunction of the alternative pathway of the complement system, which leads to the spontaneous activation of the complement system in the circulating plasma or cell surface. Recently our group published the cohort analysis of aHUS in Japan ( $n=118)$. Through the study, we revealed the followings; 1 ) the genetic background of aHUS in Japan was different from that in Western countries, 2) the most frequent genetic mutation detected in this study was I1157T in C3 $(n=24)$, which was associated with superior renal outcome in spite of frequent replases, 3) Anti-CFH antibody positive aHUS had an excellent renal outcome, 4) $44 \%$ cases presented nephrotic syndrome, 5) only $12 \%$ developed end stage renal disease (ESRD) and 6) there were 13 cases that discontinued eculizumab treatment and were followed up. These findings might help establishing the robust evidence for the optimal treatment of aHUS.

Atypical hemolytic uremic syndrome (aHUS) is a rare disease caused by the dysfunction of alternative pathway of the complement system, which leads to the spontaneous activation of the complement system in the circulating plasma or cell surface. It presents with the classic clinical triad of hemolytic anemia, thrombocytopenia and acute kidney injury, and has been reported to have a poor prognosis; it has a mortality rate of $1-7 \%$ at the first year of disease presentation, and around $30-60 \%$ of patients develop end stage renal disease (ESRD) within the 5 years of disease presentation ${ }^{1}$. Primary causes of complement regulation defect in aHUS are genetic mutations of genes encoding complement regulatory factors such as complement factor $\mathrm{H}$ (CFH) (loss of function) or complement component itself such as C3 (gain of function) (known as genetic aHUS), or the neutralizing antibodies to the regulatory factors (especially to $\mathrm{CFH}$ ) (known as acquired aHUS), all of which induce the spontaneous activation of complement alternative pathway in the patients of aHUS.

Diagnosis of aHUS is made clinically by the presence of the triad as well as an exclusion of underlying diseases causing thrombotic microangiopathy such as Shiga-toxin producing E.coli HUS (STECHUS), thrombotic thrombocytopenic purpura (TTP), autoimmune diseases, malignant hypertension, pregnancy etc. The drawback of the current diagnostic procedure of aHUS is the lack of decisive inclusion criteria.

Genetic analysis is essential for the diagnosis of the disease and 
the prognosis depends on the genetic abnormalities. To date, genetic abnormalities of chiefly 7 factors are identified to be responsible for the onset of aHUS; complement factor $\mathrm{H}(\mathrm{CFH})$, complement factor I (CFI), complement $\mathrm{C} 3$, complement factor $\mathrm{B}$ (CFB), membrane cofactor protein (MCP), thrombomodulin (THBD), and diacylglycerol kinase $\varepsilon$ (DGKE). In Western countries, relative frequencies of each affected gene are reported as below; CFH (20-30\%), CD46 (MCP) (5-15\%), CFI (4-10\%), C3 (2-10\%), CFB (1$4 \%)$ and THBD (3-5\%) $)^{1-5}$. Racial and regional differences were suggested by the previous report from Japan, which showed a high incidence of C3 variants in Japanese aHUS patients in a small cohort ${ }^{6,7}$. Recently our group reported the large cohort analysis of aHUS in Japan $(n=118)^{8}$. We revealed that the most frequent genetic abnormalities found in the Japanese cohort was C3 (31\%) with less frequency in $\mathrm{CFH}$ (10\%), which implicates that the genetic background of this disease in Japan is different from that in Western countries. In addition, majority of the mutations in C3 was c.3470T >C p.I1157T NM_000064.3 (referred to as I1157T hereinafter) $(24 / 31$ (77\%) in total C3 mutations) and surprisingly, this mutation is associated with a superior renal outcome in spite of frequent relapses ${ }^{8}$. This specific mutation resulted in lower incidences of reduced C3 levels, severe anemia and severe acute kidney injury which is represented by the renal replacement therapy required after the onset of the disease compared to the other groups. This indicates that the I1157T mutation causes less severe spontaneous complement activation. Several lines of evidence indicated that C3 mutations are generally associated with intermediate outcome. For example, 40$50 \%$ of patients with genetic abnormalities in C3 present ESRD within the five years following presentation in Western cohort, and the mutation of I1157T is quite rare in those cohorts ${ }^{1,3,9-13}$. Due to the limited number of I1157T mutation in C3 from other countries, we cannot compare the outcome and prognosis of this specific mutation among the countries.

Another distinct feature of aHUS cohort in Japan was that factor $\mathrm{H}$ autoantibody-mediated aHUS has relatively high prevalence (17\% of total aHUS) and is associated with an excellent renal outcome with no ESRD occurrence ${ }^{8}$. Although the cohort analyses of anticomplement regulatory protein autoantibody mediated aHUS are limited, $6-10 \%$ of aHUS patients are implicated to have disease causative autoantibodies ${ }^{14}$, and the prognosis of antibody-mediated aHUS was reported to be intermediate with the occurrence of ESRD at around $20-50 \%$ within the five years of presentation ${ }^{11,15,16}$. It still remains unclear if the quantitative and qualitative differences of the autoantibody accounts for the prognosis. Since not only antibody-mediated aHUS but also aHUS by CFH mutations which has the severest outcome have better outcome compared to the Western cohort, it may be possible that the severe cases were censored in our cohort study at the time of reference to our center. Many reports indicated that the antibodymediated aHUS is highly associated to the genetic abnormalities such as homozygous deletion of CFHR1 and CFHR3, which is implicated to be involved in the generation of autoantibodies ${ }^{16-19}$. Whether or not the genetic background of CFHR1 and CFHR3 (or other CFHRs etc.) which is associated with the development of antibody-mediated aHUS is different between the cohort in Japan and the other still remains to be elucidated.

The previous studies lacked the data of complete urinalyses including urinary protein excretion. Although hemoglobinuria causes positive urinary protein in urinalysis, it has not been clarified how much of urinary protein can be observed in aHUS patients. We showed almost all cases presents massive proteinuria and surprisingly $44 \%$ of total exhibited nephrotic syndrome ${ }^{8}$.

We also pointed out that the efficacy of eculizumab on ESRD, not on AKI. In spite that most aHUS cases had renal involvement with the diagnosis of AKI, ESRD, which represents severe chronic renal dysfunction, was observed in 14 cases $(12 \%)^{8}$. Of note, 4 cases (29\%) withdrew from and no longer required renal replacement therapy (hemodialysis) without kidney transplantation after the treatment of eculizumab for one year or longer. This gradual recovery of renal function might implicate that sustained inhibition of spontaneous activation of the complement system may promote intrinsic repair mechanisms of kidney. The detailed clinical profiles of this group still remain to be elucidated.

The timing to discontinue the eculizumab treatment is still open and controversial. We reported 13 cases that discontinued eculizumab treatment ${ }^{8}$. There were three major groups; C3 I1157T mutation $(n=4)$, antiCFH antibody positive $(n=5)$, unidentified etiology $(n=3)$. Surprisingly only one case in C3 I1157T mutation relapsed and the others maintained remission for 1 year or longer in most cases. Limited number of evidence from Western countries suggested that $20-30 \%$ of aHUS cases treated with eculizumab relapsed after its cessation ${ }^{20-23}$. Although the relapse rate is dependent on the genetic background of aHUS cohort and observation period etc., our data may reflect that the less severe mutation is abundant in Japan and the superior outcome of whole cohort of aHUS in Japan.

Since aHUS is a rare disease and still lacks the clinical data, effort should be paid to establish the robust evidence for the optimal treatment of aHUS. The cost to treat aHUS is so high due to repetitive eculizumab administration or plasmapheresis. Herein racial and regional differences in aHUS are emphasized, which will help establishing clinical guidelines. 


\section{Acknowledgement}

This article was published by financial support of Health Labour Sciences Research Grant from The Ministry of Health Labour and Welfare.

\section{References}

1. Fremeaux-Bacchi V, Fakhouri F, Garnier A, et al. Genetics and outcome of atypical hemolytic uremic syndrome: a nationwide French series comparing children and adults. Clin J Am Soc Nephrol. 2013; 8(4): p. 554-62.

2. Geerdink LM, Westra D, van Wijk JA, et al. Atypical hemolytic uremic syndrome in children: complement mutations and clinical characteristics. Pediatr Nephrol. 2012; 27(8): p. 1283-91.

3. Noris M, Caprioli J, Bresin E, et al. Relative role of genetic complement abnormalities in sporadic and familial aHUS and their impact on clinical phenotype. Clin J Am Soc Nephrol. 2010; 5(10): p. 1844-59.

4. Noris M, Remuzzi G. Genetic abnormalities of complement regulators in hemolytic uremic syndrome: how do they affect patient management. Nat Clin Pract Nephrol. 2005' 1(1): p. 2-3.

5. Noris M, Remuzzi G. Atypical hemolytic-uremic syndrome. N Engl J Med. 2009; 361(17): p. 1676-87.

6. Matsumoto T, Fan X, Ishikawa E, et al. Analysis of patients with atypical hemolytic uremic syndrome treated at the Mie University Hospital: concentration of C3 p.I1157T mutation. Int J Hematol. 2014; 100(5): p. 437-42.

7. Yoshida Y, Miyata T, Matsumoto M, et al. A novel quantitative hemolytic assay coupled with restriction fragment length polymorphisms analysis enabled early diagnosis of atypical hemolytic uremic syndrome and identified unique predisposing mutations in Japan. PLoS One. 2015; 10(5): p. e0124655.

8. Fujisawa $M$, Kato $H$, Yoshida $Y$, et al. Clinical characteristics and genetic backgrounds of Japanese patients with atypical hemolytic uremic syndrome. Clin Exp Nephrol. 2018.

9. Roumenina LT, Frimat M, Miller EC, et al. A prevalent C3 mutation in aHUS patients causes a direct $\mathrm{C} 3$ convertase gain of function. Blood. 2012; 119(18): p. 4182-91.

10. Schramm EC, Roumenina LT, Rybkine T, et al. Mapping interactions between complement $\mathrm{C} 3$ and regulators using mutations in atypical hemolytic uremic syndrome. Blood. 2015; 125(15): p. 2359-69.

11. Schaefer F, Ardissino G, Ariceta G, et al. Clinical and genetic predictors of atypical hemolytic uremic syndrome phenotype and outcome. Kidney Int. 2018.

12. Maga TK, Nishimura CJ, Weaver AE, et al. Mutations in alternative pathway complement proteins in American patients with atypical hemolytic uremic syndrome. Hum Mutat. 2010; 31(6): p. E1445-60.

13. Martínez-Barricarte $\mathrm{R}$, Heurich M, López-Perrote $\mathrm{A}$, et al. The molecular and structural bases for the association of complement C3 mutations with atypical hemolytic uremic syndrome. Mol Immunol. 2015; 66(2): p. 263-73.

14. Kavanagh D, Richards A, Fremeaux-Bacchi V, et al. Screening for complement system abnormalities in patients with atypical hemolytic uremic syndrome. Clin J Am Soc Nephrol. 2007; 2(3): p. 591-6.

15. Sinha A, Gulati A, Saini S, et al. Prompt plasma exchanges and immunosuppressive treatment improves the outcomes of anti-factor $\mathrm{H}$ autoantibody-associated hemolytic uremic syndrome in children. Kidney Int. 2014; 85(5): p. 1151-60.

16. Dragon-Durey MA, Sethi SK, Bagga A, et al. Clinical features of antifactor $\mathrm{H}$ autoantibody-associated hemolytic uremic syndrome. J Am Soc Nephrol. 2010; 21(12): p. 2180-7.

17. Dragon-Durey MA, Blanc C, Marliot F, et al. The high frequency of complement factor $\mathrm{H}$ related CFHR1 gene deletion is restricted to specific subgroups of patients with atypical haemolytic uraemic syndrome. J Med Genet. 2009; 46(7): p. 447-50.

18. Józsi M, Licht C, Strobel S, et al. Factor H autoantibodies in atypical hemolytic uremic syndrome correlate with CFHR1/CFHR3 deficiency. Blood. 2008; 111(3): p. 1512-4.

19. Moore I, Strain L, Pappworth I, et al. Association of factor $\mathrm{H}$ autoantibodies with deletions of CFHR1, CFHR3, CFHR4, and with mutations in $\mathrm{CFH}, \mathrm{CFI}, \mathrm{CD} 46$, and $\mathrm{C} 3$ in patients with atypical hemolytic uremic syndrome. Blood. 2010; 115(2): p. 379-87.

20. Ardissino G, Testa S, Possenti I, et al. Discontinuation of eculizumab maintenance treatment for atypical hemolytic uremic syndrome: a report of 10 cases. Am J Kidney Dis. 2014; 64(4): p. 633-7.

21. Fakhouri F, Fila M, Provôt F, et al. Pathogenic Variants in Complement Genes and Risk of Atypical Hemolytic Uremic Syndrome Relapse after Eculizumab Discontinuation. Clin J Am Soc Nephrol. 2017; 12(1): p. 50-59.

22. Merrill SA, Brittingham ZD, Yuan X, et al. Eculizumab cessation in atypical hemolytic uremic syndrome. Blood. 2017; 130(3): p. 368-372.

23. Wijnsma KL, Duineveld C, Volokhina EB, et al. Safety and effectiveness of restrictive eculizumab treatment in atypical haemolytic uremic syndrome. Nephrol Dial Transplant. 2017. 